\title{
Permanence of two species and fixed point index
}

\author{
Alfonso Ruiz-Herrera* \\ Departamento de Matemática Aplicada, \\ Facultad de Ciencias, Universidad de Granada, 18071, Spain \\ e-mail: alfonsoruiz@ugr.es
}

February 4, 2010

\begin{abstract}
We present sufficient and necessary conditions for the permanence of discrete systems in the plane based on index of fixed point on convex sets. In concrete models, a simple picture is sufficient to deduce whether our system is permanent or not.
\end{abstract}

Key words and phrases: permanence, two species, fixed points index, winding number.

\section{Introduction}

The evolution of the size of two species sharing the same habitat can be modelled by a system of difference equations of the type

$$
\left\{\begin{array}{l}
x_{n+1}=F_{1}\left(x_{n}, y_{n}\right) \\
y_{n+1}=F_{2}\left(x_{n}, y_{n}\right)
\end{array}\right.
$$

where $x_{n}, y_{n} \geq 0$ are the sizes in the period $n$. From a biological point of view it is natural to ask whether both species will coexist or if one of them will eventually disappear. The notion of permanence guarantees the coexistence of both species for any initial conditions but it carries some additional information. The system (1.1) is said to be permanent if it is possible to find two constants $0<\underline{\sigma}<\bar{\sigma}$ such that given initial conditions $x_{0}>0, y_{0}>0$ there exists $N=N\left(x_{0}, y_{0}\right)$ with

$$
\underline{\sigma} \leq x_{n} \leq \bar{\sigma}, \quad \underline{\sigma} \leq y_{n} \leq \bar{\sigma} \text { if } n \geq N .
$$

* Supported by a grant FPU 2008 and the research project 2008-02502, Ministerio de Educación y Ciencia, Spain. 
Notice that the numbers $\underline{\sigma}$ and $\bar{\sigma}$ are independent of the initial conditions.

The problem of permanence for discrete population models has been intensively studied and several techniques have been applied. Among them are averaged Lyapunov functions, Lyapunov exponents, Conley index, Morse decompositions... See for instance [3], [4], [5], [6], [8], [12], [9], [10] and the references therein. In this paper we are going to approach the problem of permanence using the fixed point index on convex sets (see section 2 or [1]). This is a topological tool usually employed in Nonlinear Analysis to prove the existence of positive solutions of certain equations and systems. One of the task of the paper will be to show how this index is naturally linked to the problem of permanence. The main advantage of the use of the index is that, being of topological nature, it can deal with non-hyperbolic situations. A second feature of our method is its geometrical flavour. In concrete examples permanence can be decided by looking at two curves which can be drawn with a computer. This can replace the more standard computations of eigenvalues needed in previous results.

Besides the fixed point index we shall employ a result by Kirchgraber and Palmer in [7]. This is a result on the dynamics of a diffeomorphism around a partially hyperbolic fixed point and its use will impose some restrictions on the range of applicability of the method. In particular we must assume that the dynamics of each species in the absence of the other is simple, namely convergence to an equilibrium. For instance, if we let $y=0$, we need to know that the solutions of the single equation

$$
x_{n+1}=F_{1}\left(x_{n}, 0\right)
$$

converge to the unique positive solution of $x=F_{1}(x, 0)$. A question to decide in future works will be whether these restrictions are essential or just due to the use of the results in [7].

The structure of the paper is as follows. In the section 2 we introduce some preliminary notions, including the fixed point index and state the main results of the paper. In the next Section we present the proofs of these results. Finally, in the Section 4 , we will show the geometric character of the method with a concrete example.

\section{Main result and the definition of fixed point in- dex}

The first quadrant of the plane will be denoted by $\mathbb{R}_{+}^{2}$. We assume that this set is closed and denote its interior by $\operatorname{Int} \mathbb{R}_{+}^{2}$. In coordinates,

$$
\mathbb{R}_{+}^{2}=\left\{(x, y) \in \mathbb{R}^{2}: x \geq 0, y \geq 0\right\}, \text { Int } \mathbb{R}_{+}^{2}=\left\{(x, y) \in \mathbb{R}_{+}^{2}: x>0, y>0\right\} .
$$

Consider a map $F: \mathbb{R}_{+}^{2} \longrightarrow \mathbb{R}_{+}^{2},(x, y) \mapsto\left(F_{1}(x, y), F_{2}(x, y)\right)$ of class $\mathcal{C}^{1}$ and satisfying

$$
\begin{gathered}
F_{1}(x, y)>0, F_{2}(x, y)>0 \text { if } x>0 \text { and } y>0, \\
F_{1}(0, y)=F_{2}(x, 0)=0 \text { for any } x, y \geq 0 .
\end{gathered}
$$


From these conditions, we deduce that, for each initial condition $\left(x_{0}, y_{0}\right) \in \mathbb{R}_{+}^{2}$, the system of difference equations

$$
\left\{\begin{array}{l}
x_{n+1}=F_{1}\left(x_{n}, y_{n}\right) \\
y_{n+1}=F_{2}\left(x_{n}, y_{n}\right)
\end{array}\right.
$$

has a well defined forward solution $\left\{\left(x_{n}, y_{n}\right)\right\}_{n \geq 0}$ lying in $\mathbb{R}_{+}^{2}$. Moreover the sets Int $\mathbb{R}_{+}^{2},\{x=0\}$ and $\{y=0\}$ are positively invariant. We will always assume that the system (1.3) is dissipative i.e. there exists a constant $M>0$ such that

$$
\limsup \left\|\left(x_{n}, y_{n}\right)\right\| \leq M
$$

for any sequence in $\mathbb{R}_{+}^{2}$ obtained from $(1.3),\left(\|\cdot\|\right.$ is a concrete norm in $\left.\mathbb{R}^{2}\right)$. This assumption is usual in populations models since it reflects the limitations of the environment. A second assumption will concern the behavior of the system near the origin. It will be assumed that there exists $V$, neighborhood of the origin in $\mathbb{R}_{+}^{2}$ such that for each $(x, y) \in \operatorname{Int} \mathbb{R}_{+}^{2}$, there exists $N_{0}=N_{0}(x, y) \geq 0$ such that $\left(x_{N_{0}}, y_{N_{0}}\right) \notin V$. When this happens we will say that the origin does not attract interior points. In particular this condition excludes the possibility of simultaneous extinction of both species. Finally we are going to impose conditions on the dynamics in the absence of one of the species. For the difference equation

$$
x_{n+1}=F_{1}\left(x_{n}, 0\right)
$$

we assume that there exists a fixed point $x_{*}$ satisfying $0<\left|\partial_{x} F_{1}\left(x_{*}, 0\right)\right|<1$ and such that $x_{n} \longrightarrow x_{*}$ for every positive solution $\left\{x_{n}\right\}_{n \geq 0}$ of (1.4). In this case we will say that $x_{*}$ is a hyperbolic attractor on the x-axis. For the equation on the $y$-axis

$$
y_{n+1}=F_{2}\left(0, y_{n}\right)
$$

we can impose an analogous condition and say that $y_{*}>0$ is a hyperbolic attractor on the $y$-axis. An alternative assumption for (1.5) will be the nonexistence of positive fixed points. Notice that the dissipativity together with the invariance of the $y$-axis imply that, in the second case, $y_{n} \longrightarrow 0$ for every solution $\left\{y_{n}\right\}$ of (1.5). Borrowing the phase portraits from the theory of continuous dynamical systems we sum up the two situations under consideration in the diagram.
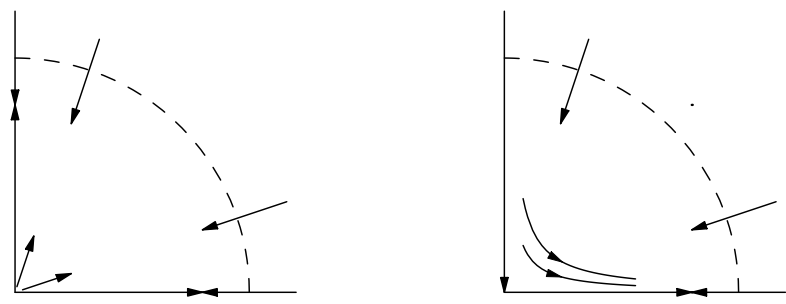

Intuitively speaking, it seems natural to expect permanence in the first case when the fixed points $\left(x_{*}, 0\right)$ and $\left(0, y_{*}\right)$ are of saddle type. For the second case, we only 
need a similar assumption on $\left(x_{*}, 0\right)$. However these fixed points are not necessarily hyperbolic. For this case, we apply the theory of the index in convex sets as developed in [1]. This theory is valid for arbitrary dimension but we only need it for $\mathbb{R}_{+}^{2}$. Next we are going to give some necessary notions on this index. First let us recall the definition of index in the whole plane $\mathbb{R}^{2}$.

Assume that $G: U: \longrightarrow \mathbb{R}^{2}$ is a continuous map defined on an open subset $U$ of $\mathbb{R}^{2}$ and having an isolated fixed point $p=G(p)$. The index can be defined as the winding number of the loop

$$
t \in[0,1] \mapsto \alpha(t)-G(\alpha(t)) \in \mathbb{R}^{2}-\{0\}
$$

where $\alpha(t)=p+\epsilon(\cos 2 \pi t, \sin 2 \pi t)$ is a positive parametrization of a small circumference centered at $p$. More precisely

$$
\operatorname{index}_{\mathbb{R}^{2}}(G, p):=\frac{\theta(1)-\theta(0)}{2 \pi}
$$

where $\theta:[0,1] \longrightarrow \mathbb{R}$ is a continuous function with

$$
\frac{\alpha(t)-G(\alpha(t))}{\|\alpha(t)-G(\alpha(t))\|}=(\cos \theta(t), \sin \theta(t)) .
$$

To define the index in $\mathbb{R}_{+}^{2}$ we start with a continuous function $F: U \cap \mathbb{R}_{+}^{2} \longrightarrow \mathbb{R}_{+}^{2}$ having an isolated fixed point at $p$. When $p$ is in the interior of $\mathbb{R}_{+}^{2}$ the definition is the same as before but when $p$ is on the boundary of $\mathbb{R}_{+}^{2}$ we define

$$
\operatorname{index}_{\mathbb{R}_{+}^{2}}(F, p):=\operatorname{index}_{\mathbb{R}^{2}}(\bar{F}, p) .
$$

Here $\bar{F}: U \longrightarrow \mathbb{R}_{+}^{2}$ is any extension of $F$ taking values on $\mathbb{R}_{+}^{2}$. Once these notions have been shown, we are ready to state our results.

Theorem 2.1 Assume that (1.3) is dissipative and the origin does not attract interior points. In addition there exist $x_{*}>0$ and $y_{*}>0$ hyperbolic global attractors for (1.4) and (1.5) respectively. Then (1.3) is permanent if only if $\operatorname{index}_{\mathbb{R}_{+}^{2}}\left(F,\left(x_{*}, 0\right)\right)=\operatorname{index}_{\mathbb{R}_{+}^{2}}\left(F,\left(0, y_{*}\right)\right)=0$.

In many models one of the species cannot survive in the absence of the other. This is the case of some prey-predator models. This motivates us to consider the following theorem.

Theorem 2.2 Assume all the conditions of the previous theorem excepting that there is no positive fixed point on the y-axis. Then (1.3) is permanent if only if $\operatorname{index}_{\mathbb{R}_{+}^{2}}\left(F,\left(x_{*}, 0\right)\right)=0$.

\section{Proof of the main Theorem}

The proof of the theorem 2.1 is separated in two parts. In the first part, we will study the local behavior of $F$ in the fixed points of the axes using the results in [7] and in the second part we will study the global behaviour using that our system is dissipative. The proof of the theorem 2.2 is totally analogous. 


\subsection{Local behavior}

In this section we are going to work with $\left(0, y_{*}\right)$, the analogous results can be obtained if we work with $\left(x_{*}, 0\right)$. Using that $F_{1}(0, y)=0$ and $F_{1}(x, y)>0, F_{2}(x, y)>0$ for all $x, y>0$, we can define the following extension to the second quadrant

$$
\widehat{F}(x, y)=\left\{\begin{array}{l}
F(x, y) \text { if } x \geq 0 \\
s \circ F \circ s(x, y) \text { if } x \leq 0
\end{array}\right.
$$

where $s$ is the symmetry respect to the $y$-axis. Before continuing, we are going to note some interesting properties of this extension. If for some $U \subset \mathbb{R}_{+}^{2},\left.F\right|_{U}$ is a homeomorphism then $\left.\widehat{F}\right|_{U \cup s(U)}$ is also a homeomorphism where $\left.F\right|_{U}$ denotes the restriction of $F$ to $U$ and in this case, $(\widehat{F})^{-1}=\widehat{F^{-1}}$. Other interesting property is $s \circ \widehat{F} \circ s=\widehat{F}$. The previous properties can be easily checked from (1.6). We will use this extension in an auxiliar way. To compute the $\operatorname{index}_{\mathbb{R}_{+}^{2}}\left(F,\left(0, y_{*}\right)\right)$, we are going to use the following map

$$
\bar{F}(x, y)=F(|x|,|y|) .
$$

Next we are going to present the essential concept to prove our theorem.

Definition 3.1 A fixed point $\left(0, y_{*}\right)$ is a weak repeller for the system (1.3) if there exists $U$ a neighborhood of $\left(0, y_{*}\right)$ in $\mathbb{R}_{+}^{2}$ so that for all $(x, y) \in U \cap$ Int $\mathbb{R}_{+}^{2}$, there is $N_{0}=N_{0}(x, y)$ such that $\left(x_{N_{0}}, y_{N_{0}}\right) \notin U$.

It is clear that if $\left(0, y_{*}\right)$ is a weak repeller for $(1.3)$ then the system is not permanent. The key in our paper is to characterize when $\left(0, y_{*}\right)$ is a weak repeller via $\operatorname{index}_{\mathbb{R}_{+}^{2}}\left(F,\left(0, y_{*}\right)\right)$.

Theorem 3.1 Assume that $\left(0, y_{*}\right)$ is a hyperbolic attractor for the system (1.3) in the $y$-axis and an isolated fixed point for $F$ in $\mathbb{R}_{+}^{2}$, then $\left(0, y_{*}\right)$ is a weak repeller if only if index $\mathbb{R}_{+}^{2}\left(F,\left(0, y_{*}\right)\right)=0$.

Before giving the proof of this result we need a preliminar result.

Lemma 3.1 Assume that $\left(0, y_{*}\right)$ is a hyperbolic global attractor in the $y$-axis.

1. If $\partial_{x} F_{1}\left(0, y_{*}\right)>1$ then index $\mathbb{R}_{+}^{2}\left(F,\left(0, y_{*}\right)\right)=0$.

2. If $\partial_{x} F_{1}\left(0, y_{*}\right)<1$ then index $\mathbb{R}_{+}^{2}\left(F,\left(0, y_{*}\right)\right)=1$.

Proof. First we assume that $\partial_{x} F_{1}\left(0, y_{*}\right)>1$. Then it is clear that there exist $\epsilon>0$ and $D$ a disk centered at $\left(0, y_{*}\right)$ such that $\partial_{x} F_{1}(x, y)>1+\epsilon$ for all $(x, y) \in D$. From these comments and using that $F_{1}(0, y)=0$, we deduce that

$$
(1+\epsilon) x<F_{1}(x, y)
$$

for all $x>0$ with $(x, y) \in D$. Next, we define the following homotopy

$$
H:[0,1] \times \bar{D} \longrightarrow \mathbb{R}^{2}
$$




$$
H(t,(x, y))=t \bar{F}(x, y)+(1-t) \widetilde{F}(x, y)
$$

where

$$
\widetilde{F}(x, y)=\left\{\begin{array}{l}
\left((-1-\epsilon) x, F_{2}(0, y)\right) \\
\left((1+\epsilon) x, F_{2}(0, y)\right) .
\end{array}\right.
$$

First, let us prove that $H$ is an admissible homotopy, i.e. $H(t,(x, y)) \neq(x, y)$ for all $(x, y) \in \partial D$. Indeed, consider $\left(x_{0}, y_{0}\right) \in \partial D$ with $x_{0} \neq 0$. From (1.7), we deduce that

$$
\left(1-t_{0}\right)(1+\epsilon)\left|x_{0}\right|+t_{0} F_{1}\left(\left|x_{0}\right|, y_{0}\right) \geq(1+\epsilon)\left|x_{0}\right|
$$

holds. For $x_{0}=0$, we deduce that $H$ does not have fixed points in $\partial D$ using that $\left(0, y_{*}\right)$ is a hyperbolic attractor. Finally using the invariance by homotopy together with product property of the index we conclude that

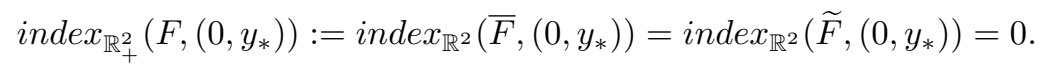

To prove the second statement, consider $\epsilon$ and $D$ analogous to the previous case and consider the map

$$
\widetilde{F}(x, y)=\left((1-\epsilon) x, F_{2}(0, y)\right) .
$$

Proof of the theorem 3.1. Firstly we are going to compute the Jacobian matrix of $F$ in $\left(0, y_{*}\right)$

$$
J_{F}\left(0, y_{*}\right)=\left(\begin{array}{cc}
\partial_{x} F_{1}\left(0, y_{*}\right) & 0 \\
\partial_{x} F_{2}\left(0, y_{*}\right) & \underbrace{\partial_{y} F_{2}\left(0, y_{*}\right)}_{\eta}
\end{array}\right) .
$$

From this expression we deduce that the eigenvalues of $J_{F}\left(0, y_{*}\right)$ are

$$
\left\{\partial_{x} F_{1}\left(0, y_{*}\right), \eta\right\} .
$$

As $\left(0, y_{*}\right)$ is a hyperbolic global attractor in the $y$-axis we deduce that $0<|\eta|<1$. On the other hand, since $F\left(\operatorname{Int} \mathbb{R}_{+}^{2}\right) \subset \operatorname{Int} \mathbb{R}_{+}^{2}$ we obtain that $\partial_{x} F_{1}\left(0, y_{*}\right) \geq 0$. In this moment we can deduce that if $\partial_{x} F_{1}\left(0, y_{*}\right)<1,\left(0, y_{*}\right)$ is an attractor and if $\partial_{x} F_{1}\left(0, y_{*}\right)>1,\left(0, y_{*}\right)$ is a weak repeller. From these comments together with the previous lemma we obtain

$$
\operatorname{index}_{\mathbb{R}_{+}^{2}}\left(F,\left(0, y_{*}\right)\right)=\left\{\begin{array}{lll}
0 & \text { if } & \partial_{x} F_{1}\left(0, y_{*}\right)>1 \\
1 & \text { if } & \partial_{x} F_{1}\left(0, y_{*}\right)<1
\end{array}\right.
$$

The rest of the proof consists in the study of the case $\partial_{x} F_{1}\left(0, y_{*}\right)=1$. Again using the expression of the Jacobian matrix, we can deduce that there exists $F^{-1}$ in a neighbourhood of $\left(0, y_{*}\right)$ and has the following expression (in this neighbourhood)

$$
F^{-1}(x, y)=\left(g(x, y), \frac{1}{\eta} y+Y(x, y)\right)
$$

where $\partial_{x} g\left(0, y_{*}\right)=1, \partial_{y} g\left(0, y_{*}\right)=0, \partial_{y} Y\left(0, y_{*}\right)=0$ and $Y$ is bounded. From these comments, we can deduce that in a neighbourhood of $\left(0, y_{*}\right), F^{-1}$ verifies the 
hypotheses $\mathbf{H}$ considered in [7], namely there exist positive constants $k_{x x}, k_{x y}, k_{y x}$, $k_{y y}$ verifying

$$
\begin{gathered}
\left|g\left(x_{1}, y_{1}\right)-g\left(x_{2}, y_{2}\right)\right| \leq k_{x x}\left|x_{1}-x_{2}\right|+k_{x y}\left|y_{1}-y_{2}\right| \\
\left|Y\left(x_{1}, y_{1}\right)-Y\left(x_{2}, y_{2}\right)\right| \leq k_{y x}\left|x_{1}-x_{2}\right|+k_{y y}\left|y_{1}-y_{2}\right|
\end{gathered}
$$

and for $\beta=\frac{1}{\eta}-k_{y y}, \alpha=k_{x x}$, there exists a constant $\rho \geq 1$ such that

$$
\begin{gathered}
\alpha<\rho<\beta \\
k_{x y} k_{y x}<(\beta-\rho)(\rho-\alpha) .
\end{gathered}
$$

To check this we consider the Lipschitz constant on $D_{r} \cap \mathbb{R}_{+}^{2}$, where $D_{r}$ is the disk centered at $\left(0, y_{*}\right)$ of the radius $r$. It is clear that

$$
k_{x x} \longrightarrow 1, k_{x y} \longrightarrow 0, k_{y y} \longrightarrow 0
$$

as $r \longrightarrow 0$ while $k_{x y}$ remains bounded. We pick a fixed $\rho>1$ lying between $\alpha$ and $\beta$ for small $r$. Then $(\beta-\rho)(\rho-\alpha)$ is a fixed quantity and the product $k_{x y} k_{y x}$ tends to zero. Next we are going to consider $\widehat{F^{-1}}(x, y)=\left(\widehat{g}(x, y), \frac{1}{\eta} y+\widehat{Y}(x, y)\right)$ where

$$
\widehat{g}(x, y)=\left\{\begin{array}{l}
g(x, y) \quad \text { if } x \geq 0 \\
-g(-x, y) \text { if } x \leq 0
\end{array}\right.
$$

and $\widehat{Y}(x, y)=Y(|x|, y)$. The map $\widehat{F^{-1}}$ is not necessary $\mathcal{C}^{1}$ but it still verifies the hypotheses $\mathbf{H}$ and so applying Theorem 1.c page 44 in [7], we deduce that $\widehat{F^{-1}}$ is topologically conjugate to

$$
G^{-1}(x, y)=\left(\widehat{g}(x, h(x)), \frac{1}{\eta} y\right)
$$

where $h:]-\epsilon, \epsilon\left[\longrightarrow \mathbb{R}\right.$ is a Lipschitz continuous function with $h(0)=y_{*}$ and $\widetilde{M}=$ $\{(x, h(x)): x \in]-\epsilon, \epsilon[\}$ is a local invariant manifold associated to $\widehat{F^{-1}}$. We notice that $\{(x, h(x)): x \geq 0\}$ is also invariant for $F^{-1}$. Therefore $\widehat{F}$ is topologically conjugate to

$$
G(x, y)=\left(\widehat{F}_{1}(x, h(x)), \eta y\right)
$$

It is posible to see that the previous map is the inverse of $G^{-1}$ in a neighbourhood of $\left(0, y_{*}\right)$ from

$$
\begin{aligned}
G(\widehat{g}(x, h(x)), & \left.\frac{1}{\eta} y\right)=\left(\widehat{F_{1}}(\widehat{g}(x, h(x)), h(\widehat{g}(x, h(x)))), y\right)= \\
= & \left(\widehat{F_{1}}\left(\widehat{F^{-1}}(x, h(x))\right), y\right)=(x, y)
\end{aligned}
$$

where first we have used that $\widetilde{M}=\{(x, h(x)): x \in]-\epsilon, \epsilon[\}$ is a local invariant manifold and in the second equality $\left(\widehat{F^{-1}}\right)^{-1}=\widehat{F}$. On the other hand, the homeomorphism of conjugation which it is built in $[7]$ verifies that $P(\{x=0\}) \subset\{x=0\}$ 
(See page 43 in [7]) and thus using that $s \circ \widehat{F} \circ s=\widehat{F}$, we can take $P$ verifying that $s \circ P=P \circ s$. Finally, since

$$
\bar{F}(x, y)=\left\{\begin{array}{l}
\widehat{F}(x, y)=F(x, y) \text { if } x \geq 0 \\
\widehat{F} \circ s(x, y) \text { if } x \leq 0
\end{array}\right.
$$

we deduce that $\bar{F}$ is topologically conjugate to

$$
\widetilde{G}(x, y)=\left\{\begin{array}{l}
\left(F_{1}(x, h(x)), \eta y\right) \text { if } x \geq 0 \\
\left(F_{1}(-x, h(-x)), \eta y\right) \quad \text { if } x \leq 0 .
\end{array}\right.
$$

All these comments enables us to deduce that $\left(0, y_{*}\right)$ is a weak repeller for $F$ if only if $x<F_{1}(x, h(x))$ for $x>0$. In this case, using the invariance of the index by conjugation (See Remark 14 [1]) we deduce that

$$
\operatorname{index}_{\mathbb{R}_{+}^{2}}\left(F,\left(0, y_{*}\right)\right):=\operatorname{index}_{\mathbb{R}^{2}}\left(\bar{F},\left(0, y_{*}\right)\right)=\operatorname{index}_{\mathbb{R}^{2}}(\widetilde{G},(0,0))=0
$$

and the proof is complete.

In the proof of the theorem 2.1 we will need a consequence of the above proof. If $\operatorname{index}_{\mathbb{R}_{+}^{2}}\left(F,\left(0, y_{*}\right)\right)=0$ then there exists $\delta>0$ so that for all $(x, y) \in U \cap \operatorname{Int} \mathbb{R}_{+}^{2}$, there exists $N_{0}=N_{0}(x, y)$ so that $\left(x_{N_{0}}, y_{N_{0}}\right) \notin U$ and $x_{N_{0}}>\delta$ where $U$ is the same neighbourhood provided by theorem 3.1. This can be easily seen using the Hartman-Grossman theorem (see [11]) when $\partial_{x} F_{1}\left(0, y_{*}\right) \neq 1$ and using that $\bar{F}$ is topologically conjugate to $\widetilde{G}$ when $\partial_{x} F_{1}\left(0, y_{*}\right)=1$. We can obtain the analogous conclusion for $\left(x_{*}, 0\right)$.

Remark 3.1 Note that under the hypotheses of the theorem 3.1, the posibles values

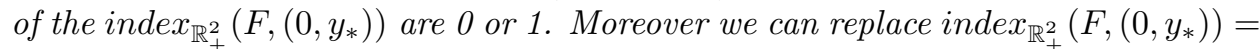

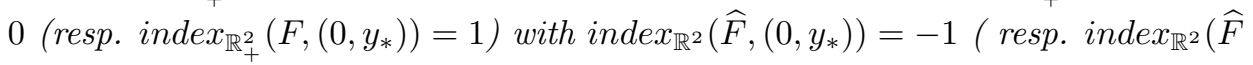
,$\left.\left.\left(0, y_{*}\right)\right)=1\right)$.

\subsection{Conclusion}

In this section we will pass from the local to the global behaviour using that our system is dissipative. To this aim it is essential the following result.

Lemma 3.2 (Lemma 2.1 in [5]) Let $F: X \longrightarrow X$ be continuous map where $X$ is a metric space and assume that $K$ is a compact set verifying that for all $x \in X$ there exists $N=N(x)$ such that $x_{N} \in K$. Then there exists $k_{0}$ such that $\widetilde{K}=\bigcup_{j=0}^{k_{0}} F^{j}(K)$ is compact and positively invariant.

From this lemma it is easy to check that our system is permanent. Indeed, since $F\left(\operatorname{Int} \mathbb{R}_{+}^{2}\right) \subset \operatorname{Int} \mathbb{R}_{+}^{2}$ and $\left(x_{*}, 0\right),\left(0, y_{*}\right)$ are hyperbolic attractors in each axis we deduce that $F^{-1}(\{0\})=0$. Using that (1.3) is dissipative and the origin does not attract interior points, we deduce that there exist $R>r>0$ so that for all $(x, y) \in$ 
$\mathbb{R}_{+}^{2} \backslash\{0\}$ there exists $N_{0}=N_{0}(x, y)$ such that $\left(x_{N_{0}}, y_{N_{0}}\right) \in K=\left(\overline{B_{R}(0)} \backslash B_{r}(0)\right) \cap \mathbb{R}_{+}^{2}$. Then applying the lemma 3.2 with $X=\mathbb{R}^{2} \backslash\{0\}$, we conclude that there exists $m_{0} \in \mathbb{N}$ such that $\bigcup_{j=0}^{m_{0}} F^{j}(K)=K_{1}$ is compact and positively invariant (notice that $0 \notin K_{1}$ ). From this moment, we are going to concentrate on $K_{1}$ to study the dynamics. Next, we are going to "separate" from the $x$-axis in the following way. Using that $\left(x_{*}, 0\right)$ is a weak repeller, we deduce that there exists $V$ a neighbourhood of $\left(x_{*}, 0\right)$ and $\delta>0$ so that for all $(x, y) \in V$ with $y>0$, there is $N_{1}=N_{1}(x, y)$ such that $\left(x_{N_{1}}, y_{N_{1}}\right) \notin V$ with $y_{N_{1}}>\delta$. Now, since $K_{1} \cap\{y=0\}$ is compact, we deduce that there exists $N_{2}$ such that for all $(x, 0) \in K_{1}$, there exists an index $j \leq N_{2}$ so that $\left(x_{j}, 0\right) \in V$. From the continuity of $F$ we deduce that there exists $\delta_{1}>0$ with $\delta>\delta_{1}$ so that for all $(x, y) \in K_{1}$ with $y \leq \delta_{1}$, there exists an index $j \leq N_{2}$ such that $\left(x_{j}, y_{j}\right) \in V$. Therefore, taking $\widetilde{K_{1}}=K_{1} \cap\{(x, y): y>\delta\}$ and again applying the lemma 3.2 , we deduce that there exists $s_{0}$ such that $K_{2}=\bigcup_{j=0}^{s_{0}} F\left(\widetilde{K_{1}}\right)$ is compact and positively invariant.

The result is concluded repeating the same argument with the $y$-axis and $K_{2}$.

\section{Applications}

The use of the index allows to deal with degenerate cases which can not be treated via hyperbolicity. However, even in the hyperbolic case, it has some interest since it replaces an algebraic computations of eigenvalues by the study of the winding number that can be done visually. To show this we start with a concrete example. Consider the model

$$
\left\{\begin{array}{l}
x_{n+1}=x_{n} \exp \left(0.5-x_{n}-4 y_{n}\left(y_{n}-1\right)\right) \\
y_{n+1}=y_{n} \exp \left(1.5-3 x-y_{n}\right)
\end{array}\right.
$$

The function $F$ satisfies all the assumptions in Theorem 2.1. To check the dissipativity we notice that $F\left(\mathbb{R}_{+}^{2}\right)$ is bounded. It is also clear that the origin does not attract interior points since the eigenvalues of the Jacobian matrix of $F$ at $(0,0)$ are $\{\exp 0.5, \exp 1.5\}$. A simple study on the axes enables us to deduce that $(0.5,0)$ and $(0,1.5)$ are hyperbolic attractors in the $x$-axis and $y$-axis respectively, (this study will be done in the next example). Therefore, we have just to compute index ${\mathbb{\mathbb { R } _ { + } ^ { 2 }}}_{(}(F,(0.5,0))$ and index $\mathbb{R}_{+}^{2}(F,(0,1.5))$. Apart from the fixed points on the axes, $\left(\frac{5}{36}, \frac{13}{12}\right)$ is the unique fixed point of our system in $\operatorname{Int}\left(\mathbb{R}_{+}^{2}\right)$. After this remark, we draw the curves $\beta_{i}(t)=\alpha_{i}(t)-\bar{F}\left(\alpha_{i}(t)\right)$ for $\alpha_{1}(t)=(0.5+$ $0.1 \cos (2 \pi t), 0.1 \sin (2 \pi t)), \alpha_{2}(t)=(0.2 \cos (2 \pi t), 1.5+0.2 \sin (2 \pi t))$ and $\bar{F}(x, y)=$ $F(|x|,|y|)$.

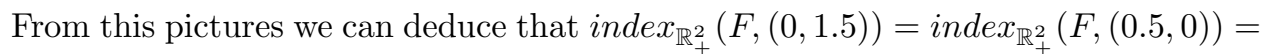
1. By the theorem 2.1 we conclude that the system is not permanent. We notice 1 is an eigenvalue of the Jacobian matrix of $F$ at $(0.5,0)$.

\section{A generalized Lotka Volterra model}


$\beta_{1}(t)$

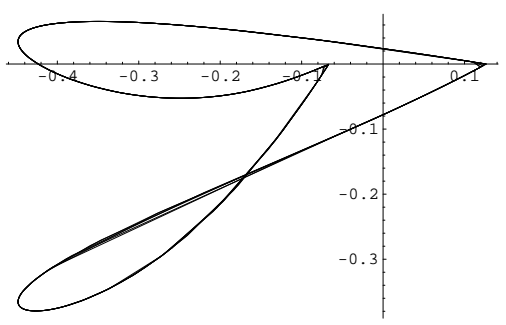

$\beta_{2}(t)$

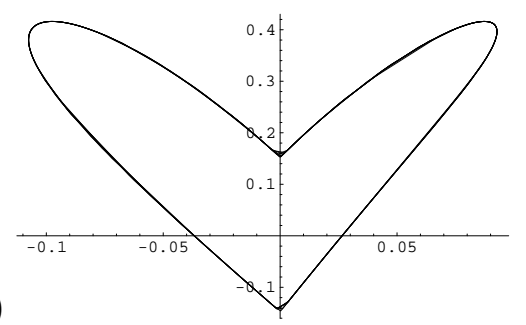

Next we consider the following model, namely

$$
\left\{\begin{array}{l}
x_{n+1}=x_{n} \exp \left(r_{1}-x_{n}-f\left(y_{n}\right)\right) \\
y_{n+1}=y_{n} \exp \left(r_{2}-g\left(x_{n}\right)-y_{n}\right)
\end{array}\right.
$$

with $\left.r_{i} \in\right] 0,2\left[\backslash\{1\}\right.$. The functions $f, g$ are of $\mathcal{C}^{1}([0, \infty[)$ with $f(0)=g(0)=0$ and for some constant $M>0, f(t), g(t) \geq 0$ for all $t \geq M$. It is known that if $0<r_{1}<1$ then $\left(x_{n}, 0\right) \longrightarrow\left(r_{1}, 0\right)$ for all $\left.x_{0} \in\right] 0,+\infty\left[\right.$ and the sequence $\left\{x_{n}\right\}$ is monotone. For $\left.r_{1} \in\right] 1,2[$, we obtain the same conclusion but in this case the sequence is oscillating (see [2]). Let us apply the theorem 2.1 to characterize the permanence of the system (1.9) under the hypotheses $\left.r_{i} \in\right] 0,2[\backslash\{1\}$.

Again using that the map is bounded we deduce that the system is dissipative. On the other hand, using that $\left\{\exp \left(r_{1}\right), \exp \left(r_{2}\right)\right\}$ are the eigenvalues of the Jacobian matrix of $F$ at $(0,0)$, we deduce that the zero does not attract interior points. Then by the Theorem 2.1 our system is permanent if only if $i n d e x_{\mathbb{R}_{+}^{2}}\left(F,\left(r_{1}, 0\right)\right)=$ $\operatorname{index}_{\mathbb{R}_{+}^{2}}\left(F,\left(0, r_{2}\right)\right)=0$. Next we are going to study when both indices are zero. Indeed, let us concentrated on the index $_{\mathbb{R}_{+}^{2}}\left(F,\left(r_{1}, 0\right)\right)$, the analogous conclusion can be obtained in the other case. Using an analogous of the lemma 3.1 for the $x$-axis, we deduce that for $r_{2}-g\left(r_{1}\right)>0$,

$$
\operatorname{index}_{\mathbb{R}_{+}^{2}}\left(F,\left(r_{1}, 0\right)\right)=0
$$

and for $r_{2}-g\left(r_{1}\right)<0$,

$$
\operatorname{index}_{\mathbb{R}_{+}^{2}}\left(F,\left(r_{2}, 0\right)\right)=1 .
$$

From this moment we concentrate on the $i n d e x_{\mathbb{R}_{+}^{2}}\left(F,\left(r_{1}, 0\right)\right)$ when

$$
r_{2}=g\left(r_{1}\right)
$$

In the rest of the argument we are going to assume that $1-f^{\prime}(0) g^{\prime}\left(r_{1}\right) \neq 0$. This condition implies that $\left(r_{1}, 0\right)$ is an isolated fixed point for $(1.9)$ since if $\left(x_{*}, y_{*}\right)$ is an fixed point with $y_{*}>0$, then

$$
\left\{\begin{array}{l}
r_{1}=x_{*}+f\left(y_{*}\right) \\
r_{2}=g\left(x_{*}\right)+y_{*}
\end{array}\right.
$$


Now we consider the curve

$$
\beta(t)=\alpha(t)-\bar{F}(\alpha(t))
$$

where $\bar{F}(x, y)=F(|x|,|y|)$ and $\alpha(t)=\left(r_{1}+\rho \cos 2 \pi t, \rho \sin 2 \pi t\right)$ for a sufficiently small $\rho>0$ and $t \in[0,1]$. Observe that $\beta(t) \in\{y<0\}$ for $t \in] \frac{1}{2}, 1\left[\right.$. Since $r_{1}$ attracts the $x$-axis, $\beta(0)=\beta(1)=\left(\xi_{1}, 0\right), \beta\left(\frac{1}{2}\right)=\left(-\xi_{2}, 0\right)$ with $\xi_{i}>0$. Next we concentrate on studying the behavior of $\beta(t)$ for $t \in] 0, \frac{1}{2}[$. In this moment we are going to distinguish two cases:

- $1-f^{\prime}(0) g^{\prime}\left(r_{1}\right)>0$,

- $1-f^{\prime}(0) g^{\prime}\left(r_{1}\right)<0$.

In the first case we can deduce that $G(y)=y+g\left(r_{1}-f(y)\right)$ is strictly increasing in a neighbourhood of $y=0$. This condition implies that for $t \in] 0, \frac{1}{2}[$, the curve $\beta(t)$ only cuts the $y$-axis in the positive part. Indeed, if for some $\left.t_{*} \in\right] 0, \frac{1}{2}[$,

$$
r_{1}-\alpha_{1}\left(t_{*}\right)-f\left(\alpha_{2}\left(t_{*}\right)\right)=0
$$

then $r_{2}-g\left(\alpha_{1}\left(t_{*}\right)\right)-\alpha_{2}\left(t_{*}\right)=r_{2}-\left(\alpha_{2}\left(t_{*}\right)+g\left(r_{1}-f\left(\alpha_{2}\left(t_{*}\right)\right)\right)\right)=r_{2}-G\left(\alpha_{2}\left(t_{*}\right)\right)<$ $r_{2}-G(0)=r_{2}-g\left(r_{1}\right)=0$, here we have used that $\alpha_{2}\left(t_{*}\right)>0$ and (1.10). In the second situation we have that for $t \in] 0, \frac{1}{2}$ [ the curve $\beta(t)$ only cuts the $y$-axis in the negative part. From this information we can deduce that index $x_{\mathbb{R}_{+}^{2}}\left(F,\left(r_{1}, 0\right)\right)=1$ in the first case and index $\mathbb{R}_{+}^{2}\left(F,\left(r_{1}, 0\right)\right)=0$ in the second one. These conclusions are clear from the following lemma.

Lemma 4.1 Let $\beta:[0,1] \longrightarrow \mathbb{R}^{2} \backslash\{0\}$ be a continuous map with $\beta(0)=\beta(1)$ and verifying,.

1. $\beta(0)=\left(\xi_{1}, 0\right)$ with $\xi_{1}>0$

2. $\beta(t)$ only cuts the $y$-axis in the positive part for $t \in\left[0, \frac{1}{2}[\right.$

3. $\beta\left(\frac{1}{2}\right)=\left(-\xi_{2}, 0\right)$ with $\xi_{2}>0$

4. $\beta(t) \in\{y<0\}$ for $t \in] \frac{1}{2}, 1[$.

Then $\frac{\theta(1)-\theta(0)}{2 \pi}=1$ where $\theta(t)$ is any continuous argument of $\beta(t)$. Moreover if we replace positive part by negative part in the condition 2$)$, we obtain $\frac{\theta(1)-\theta(0)}{2 \pi}=0$.

Proof. Take $\theta:[0,1] \longrightarrow \mathbb{R}$ a continuous argument for $\beta(t)$. Using the condition 1 ), it is not restrictive to assume that $\theta(0)=0$. After that, from the condition 2) we deduce that $\theta(t) \in] \frac{-\pi}{2}, \frac{3 \pi}{2}[$ for $t \in] 0, \frac{1}{2}$ [. In this situation the condition 3 ) implies that $\theta(t) \in] \pi, 2 \pi[$. Hence $\theta(t) \in] \pi, 2 \pi[$ if $t \in] \frac{1}{2}, 1[$ what enables us to conclude that $\theta(1)=2 \pi$.

Finally we deduce the values of the index using the definition via the winding number.

\section{Acknowledgements}

I wish thank to my advisor, professor R. Ortega, for suggesting me this kind of results using [7] and for his help in writing this paper. 


\section{References}

[1] E. N. Dancer, Degree theory on convex sets and applications to bifurcation. Calculus of variations and partial differential equations, (Pisa, 1996) SpringerVerlag, 2000.

[2] P. Cull, Local and global stability for populations models, Biological Cybernetics 54, (1989) 930-938.

[3] B. M. Garay and J. Hofbauer, Robust permanence for ecological differential equations, minimax and discretizations, SIAM J. Math. Anal. 34 (2003), 10071039 .

[4] M. W. Hirsch, H. L. Smith and X.-Q. Zhao, Chain transitivity and strong repellors for semidynamical system, J. Dynamics and Differential Equations 13 (2001), 107-131.

[5] J. Hofbauer, V. Hutson and W. Jansen, Coexistence for systems governed by difference equations of Lotka-Volterra type, J. Math. Biol. 25 (1987), 553-570.

[6] V. Hutson, A theorem on average Liapunov functions, Monash Math 98 (1984), 267-275.

[7] U. Kirchgraber and K. J. Palmer, Geometry in the neighbourhood of invariant manifold of maps and flows and linearization. Pitman Research. Notes in Mathematics Series, 233.

[8] R. Kon, Permanence of discrete-time Kolmogorov systems for two species and saturated fixed points, J. Math. Biol. 48 (2004), 57-81.

[9] Z. Lu and W. Wang, Permanence and global attractivity for Lotka-Volterra difference systems, J. Math. Biol. 39 (1999), 269-282.

[10] P. Margal and X.-Q. Zhao, Global attractors and steady states for uniformly persistent dynamical systems, SIAM J. Math. Anal. 37 (2005), 251-275.

[11] J. Palis and W. de Melo, Geometric theory of dynamical systems: An introduction. Springer- Verlag, New York- Berlin, 1982.

[12] P. Salceanu and H. L. Smith, Lyapunov exponents and the persistence in discrete dynamical systems, Discrete and Cont. Dyn. Syst. Serie B 12 (2009),187203. 